I nvest i gat i on of i mpressi ons for approach not i on of a nobile robot based on psychophysi ol ogi cal anal ysi s

\begin{tabular}{|c|c|}
\hline 著者 & $\begin{array}{l}\text { HANAJI MA Naohi ko, OHTA Youhei, H KTA } \\
\text { H r omi t su, YANASH TA M t suhi sa }\end{array}$ \\
\hline $\begin{array}{l}\text { j our nal or } \\
\text { publ i cat i on } \mathrm{title}\end{array}$ & $\begin{array}{l}2005 \text { I EEE I nt er nat i onal Wbr kshop on Robot and } \\
\text { Human I nt er act i ve Communi cat i on ( RO NAM) }\end{array}$ \\
\hline page $r$ ange & $79-84$ \\
\hline year & 2005-08 \\
\hline URL & ht t p: //hdl . handl e. net /10258/279 \\
\hline
\end{tabular}


I nvest i gat i on of i mpressi ons for approach not i on of a nobile robot based on psychophysi ol ogi cal anal ysi s

\begin{tabular}{|c|c|}
\hline 著者 & $\begin{array}{l}\text { HANAJI MA Naohi ko, OHTA Youhei, H KTA } \\
\text { H r omi t su, YANASH TA M t suhi sa }\end{array}$ \\
\hline $\begin{array}{l}\text { j our nal or } \\
\text { publ i cat i on } \mathrm{title}\end{array}$ & $\begin{array}{l}2005 \text { I EEE I nt er nat i onal Wbr kshop on Robot and } \\
\text { Human I nt er act i ve Communi cat i on ( RO NAM) }\end{array}$ \\
\hline page $r$ ange & $79-84$ \\
\hline year & 2005-08 \\
\hline URL & ht t p: //hdl . handl e. net /10258/279 \\
\hline
\end{tabular}




\title{
Investigation of impressions for approach motion of a mobile robot based on psychophysiological analysis*
}

\author{
Naohiko Hanajima, Youhei Ohta, Hiromitsu Hikita and Mitsuhisa Yamashita \\ Dept. of Mechanical Systems Engineering \\ Muroran Institute of Technology \\ Muroran, Hokkaido JAPAN 050-8585 \\ Email: hana@mondo.mech.muroran-it.ac.jp
}

\begin{abstract}
This paper investigates impressions of approach motions of a mobile robot based on psychophysiological analysis. In one of our previous studies, we suggested that actuation noise caused by the robots tended to raise the sympathetic nervous system (SNS) response of heart rate variability. In another experiment it was observed that blocking out either the sound or the sight of the robot motion attenuated the electrodermal activity (EDA), which reflects the SNS. From these investigations, one candidate for motion rules for humanfriendly robots was deduced such that robots must reduce their motion speed in the immediate vicinity of human.

To confirm validity of the motion rule, we constructed an experimental setup with a mobile robot approaching humans at several speeds, and investigated the human impressions by means of psychophysiological methods. The experimental results showed that robot motion adjacent to humans tended to increase EDA responses.

We found especially that the approach motion tented to give stronger stimuli to humans than motions at a distance. The faster approach motion tended to increase EDA responses, but there was no significant statistical difference from the response to slower approach motion. From factor analysis of the subjective ratings two factors were extracted, which were interpreted as "relief of mind" and "observation on motion."

Index Terms-mobile robot, human-friendly, motion rule, skin conductance response, subjective rating
\end{abstract}

\section{INTRODUCTION}

These days there is increased development of humanfriendly robots such as home robots or service robots. The improvement in technologies of mechatronics and computerizations turns such developments into reality. The diffusion of such robotic devices is making the space between robots and humans narrower. In industrial applications, the robots are isolated from humans to ensure the humans' safety [1]. However, where the work space of humans and robots overlap, it is important to consider the safety of the users both physically and mentally.

From the point of the view of safety, much research has been done. For the physical safety of humans collisions with robots must be avoided. Some research has been devoted to the prediction of collisions, such as a model-based approach

\footnotetext{
* This work is partially supported by Satellite Venture Business Laboratory in Muroran Institute of Technology.
}

[2] or a sensor-based approach [3], [4]. When a collision does happen, it is necessary to minimize pain and injury to the human. One approach is to give elasticity to the robots, as with a viscoelastic cover [5] or mechanical springs and dampers[6]. Investigations of safety issues in robot control have used the human pain tolerance limit[5] or introduced a danger index [7]. Such an index considers a cost function of safety and optimizes it [8]. On the issue of mental safety or stress of a human facing a moving robot, some analytical studies have described human responses using subjective evaluations, such as a rating scale [9], and physiological data, such as galvanic skin reflex [10] or pupillary dilation [11].

Motions of robotic devices give stimulus to humans mainly through auditory and visual modalities. Our previous work indicated that actuation noise from the robots tended to raise sympathetic nervous system (SNS) response of heart rate variability (HRV) [12]. In another experiment we used electrodermal activity (EDA) to evaluate SNS response [13] because the HRV analysis of data in the frequency domain claimed data acquisition for more than 25 seconds. Alternatively, the signals related to EDA respond to the stimulus instantaneously. The experiment was performed under three conditions to control influences of auditory and visual modalities: with sound blocked out, with sight blocked out, and with no blocking. The experimental results confirmed that blocking out either sound or sight attenuated the EDA responses to robot motion. From these psychophysiological investigations, a simple rule for the motion of human-friendly robots could be derived.

When the robot moved rapidly and dynamically, the noise and visual stimuli from its motion tended to be louder and larger respectively. This could lead to a simple consequence: namely, to prevent increased SNS response of a user of a human-friendly robot, the robot should move slowly in the immediate vicinity of the user. We constructed an experimental setup with a mobile robot approaching humans at several speeds, then investigated human impressions by means of psychophysiological methods to confirm whether the motion rule was valid or not. The scheme of the approach motions was as follows. A vision system installed on the mobile robot detected a skin color area, regarded as a human face, 


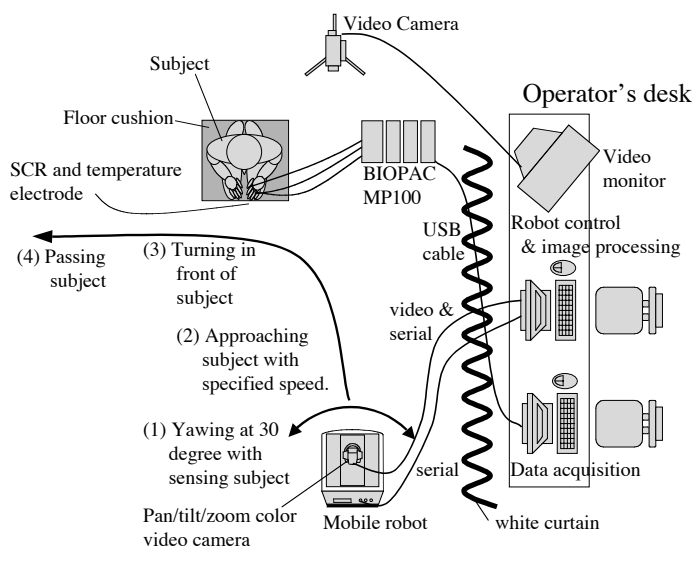

Fig. 1. Top view of experimental setup

then the robot would turn toward the area. To investigate users' impressions, the skin conductance response (SCR) of subjects was analyzed by analysis of variance (ANOVA), and subjective rating was analyzed by factor analysis.

\section{EXPERIMENT}

\section{A. Objective}

As mentioned in the Introduction, in our previous work it was observed that blocking out either the sound or the sight attenuated the EDA responses to the robot motion. It seems that this conclusion corresponds with our ordinary sense that loud noise or quick movement in close proximity is stressful to humans. We can apply this observation to robot motion generation to increase user comfort. One candidate for a rule of robot motion generation is as follows: robots must reduce their motion speed in the immediate vicinity of humans, making their noise lower and their movement slower. This rule is not only concise but also convenient for practical use. In the following section we apply this rule to a mobile robot and investigate the human impression by means of both of the SCR responses and the subjective ratings to confirm whether the rule is valid or not.

\section{B. Experimental setup}

Fig.1 shows the top view of the experimental setup. A twowheeled differential-drive mobile robot (LABO-3, AAI) was engaged in this experiment. A color video camera with remote pan/tilt/zoom operation (EVI-D100, Sony) was mounted on the mobile robot. The center of panning of the camera was aligned above the center of the axle between the robot's wheels. Images from the video camera were captured and processed by an image processing board (IP-5005, Hitachi) installed in a personal computer (Pentium 266MHz, Windows NT). The video camera could be controlled by the personal computer with VISCA commands (Sony) via serial communication. Although the mobile robot could move autonomously
TABLE I

ADJETIVE PAIRS FOR QUESTIONNAIRES

\begin{tabular}{|c||rll|}
\hline No. & negative & & positive \\
\hline \hline 1 & tensional & $\leftrightarrow$ & relaxed \\
\hline 2 & unpleasant & $\leftrightarrow$ & pleasant \\
\hline 3 & anxious & $\leftrightarrow$ & secure \\
\hline 4 & near & $\leftrightarrow$ & far \\
\hline 5 & fast & $\leftrightarrow$ & slow \\
\hline
\end{tabular}

using a microcomputer board, its computational ability was too poor to process the image data. Therefore the mobile robot was remotely controlled through a serial cable by the personal computer with the image processing board installed.

The motion of the robot was monitored from a higher place by a surveillant video camera and displayed on a monitor screen on an operator's desk for safety's sake. An operator can order the robot to stop immediately when an unexpected motion of the robot is observed on the screen.

\section{Method}

1) Subjects: Subjects were seven male volunteers aged 21-38 years old. They were involved in mechanical engineering and familiar with the robotic device. They gave informed consent to take part in an experiment. The robot motion and the procedure of the experiment were explained to them, including that the robot would not collide with them nor move so fast that they could escape it easily in the unlikely event of an impending collision. They were asked to fill in a questionnaire before the experiment.

During the experiment, each subject sat on a floor cushion and watched a mobile robot in a room measuring $4 \times 6 \mathrm{~m}$ $(13 \times 20$ feet $)$, with temperature around 22 degree Celsius. The subject and operators were separated by white curtain partitions.

2) Robot movement: The movement of the mobile robot is shown in Fig.1 by a solid line with arrow. Initial position of the robot was about two meters from the subject. Before moving, the robot searched for an object with a color equivalent to the skin color of average Japanese people, using a video camera at wide angle zoom. During the experiment, the camera tracked the object in the center of the camera image by controlling its pan and tilt. Then the mobile robot started yawing at 30 degrees on the spot repeatedly (designated as (1)). The yawing motion was introduced to accustom a subject to the robot movement itself.

About 30 seconds later, the robot began to approach the subject at a specific speed (designated as (2)). Two kinds of speed, "slow" and "fast," were prepared and tested. Upon getting close to the subject (around $55 \mathrm{~cm}$ away), the robot turned to the left in front of the subject (designated as (3)), and passed the subject (designated as (4)). 

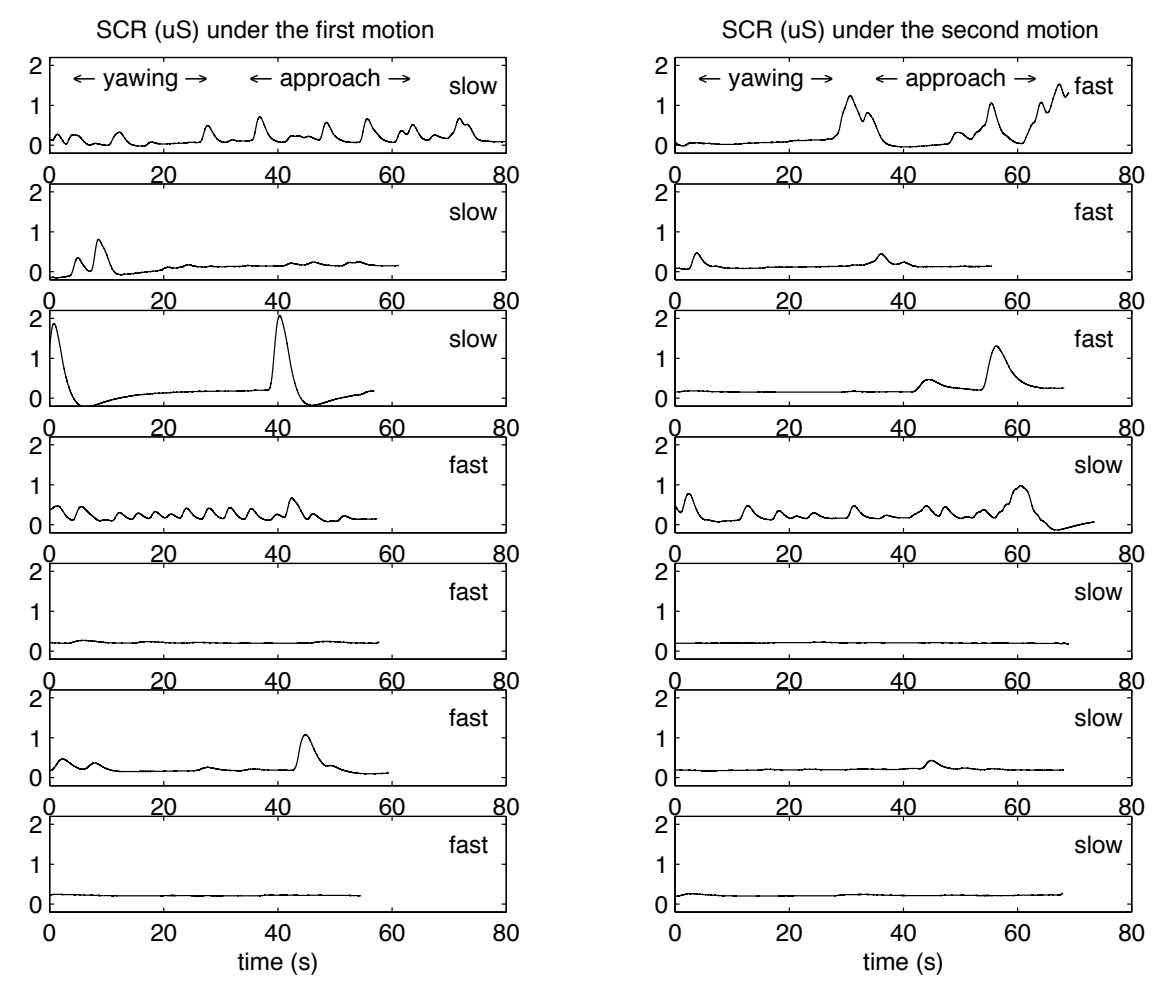

Fig. 2. SCR waves measured in this experiment

3) Measurement: The SCR was measured using a physiological measuring equipment system (MP100ACE/UMI100C/GSR100C,BIOPAC), filtered through a lowpass filter with a cut-off frequency of $1.0 \mathrm{~Hz}$ and a highpass filter with a cut-off frequency of $0.05 \mathrm{~Hz}$, and recorded onto a personal computer (IBM PC-AT compatible) via USB interface. An electrode was placed on the index finger of the subject, and one on the middle finger. The data acquisition software on the PC managed, transformed and displayed the measured data. At the same moment the skin temperature of the annular finger was measured by a temperature transducer (TSD202D/SKT100C,BIOPAC) because the subject whose skin temperature was low tended to respond with lower SCR.

4) Questionnaires: To determine the condition of the subjects a questionnaire was prepared that asked hours of sleep, ingesta and health questions. In addition, to investigate the subjects' responses to the approach motion of the robot, another questionnaire was employed using five pairs of adjectives (Table I) and analyzed by the semantic differential technique. The subjects rated their impressions according to the seven-grade system. In the statistical analysis, ratings for the positive side of the pair were assigned positive values by the three grades, ratings for the negative side were assigned negative values, and neutral ratings equaled zero.

5) Procedures: First the questionnaire and general instructions on the experiment were given to a subject. The subject was then asked to fill out an interview sheet about his physical condition. The subject sat on a floor cushion in front of the mobile robot. Electrodes to measure SCR and skin temperature were attached to appropriate fingers of the subject and physiological measurement devices were calibrated. After the stable SCR wave was confirmed and devices calibrated, the data acquisition was started as the operator triggered the robot motion. The subject was asked to keep watching the mobile robot during the measurements. When the robot stopped moving and the instantaneous response of the SCR had settled, the subjects were asked to evaluate their feelings on scales of five adjectives. This procedure was repeated once each for two kinds of robot speed. To prevent an influence of habituation in the statistical analysis, the order of the speeds was changed for each subject.

6) Data analysis: Magnitude of SCR was measured as the amplitude from base line to peak of positive wave [14]. Frequency of SCR was measured as the number of SCR waves within a specified period. The time series data for one procedure was divided at the moment between yawing and approach motions. For each period, which was around 30 seconds, the magnitude and frequency of the SCR were evaluated.

To compare averages of the SCR magnitude, the SCR frequency, and the subjective ratings, the ANOVA was performed with robot speed, order of motion, and order of measurements as independent variables [15].

To extract meaningful factors from the subjective ratings, 


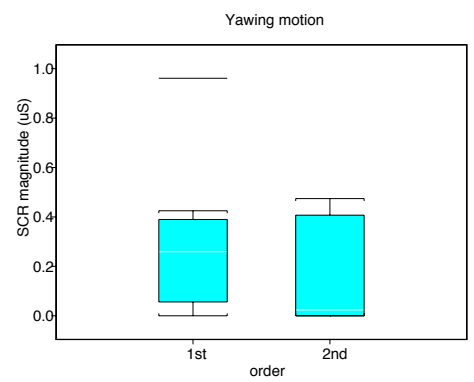

Fig. 3. Box-and-whisker plots of the SCR magnitude under the yawing motion with respect to presented order.

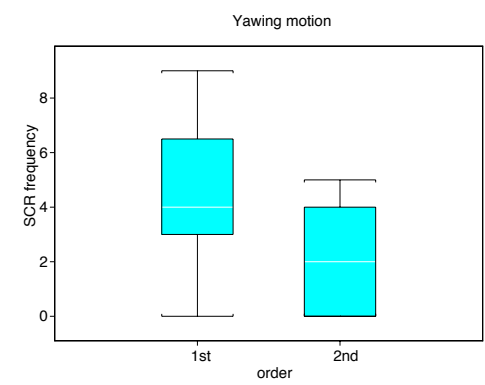

Fig. 6. Box-and-whisker plots of the SCR frequency the yawing motion with respect to presented order.

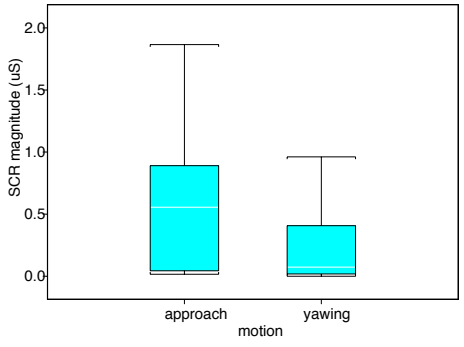

Fig. 4. Box-and-whisker plots of the SCR magnitude with respect to robot motions.

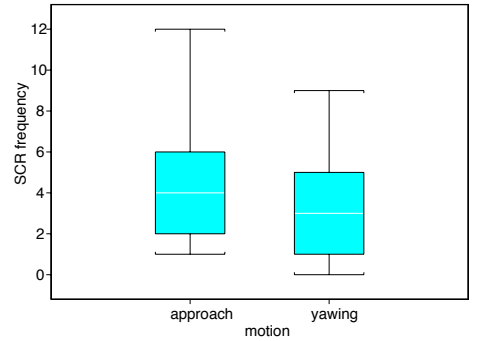

Fig. 7. Box-and-whisker plots of the SCR frequency with respect to robot motions.

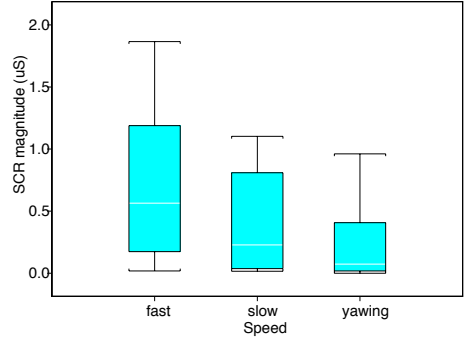

Fig. 5. Box-and-whisker plots of the SCR magnitude with respect to motion speed.

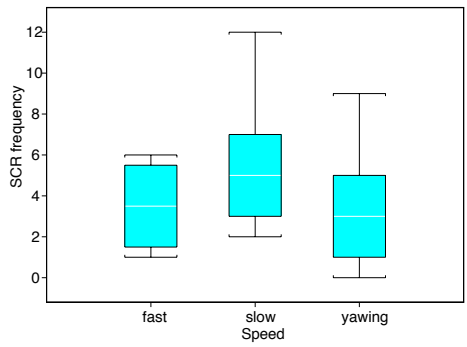

Fig. 8. Box-and-whisker plots of the SCR frequency with respect to motion speed.

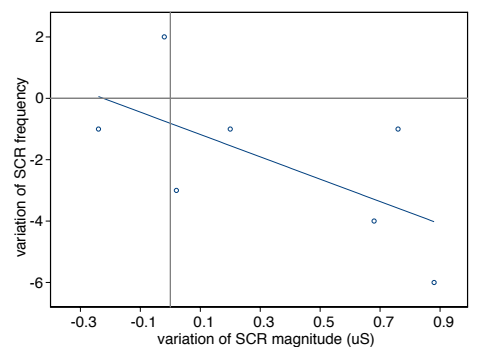

Fig. 9. Relation between variations of the SCR indices with respect to the approach speed.

factor analysis was carried out. The factors were compared with other indices.

\section{Results}

Fig. 2 shows the time series data of the SCR wave. The graphs on the left side are measurements of the first motion, and on the right side the second motion. The graphs on each row represent measurements from one subject. In the first half of the measurement period the yawing motion was presented to the subject, and in the second half the approach motion with specified speed was presented. The kind of speed is indicated on the upper right corner of the graph.

Several kinds of box-and-whisker plots are displayed in Fig.3-8. Each graph on the upper row shows the SCR mag- nitude, the lower row the SCR frequency. The graphs in the left column are categorized into the orders of the presented motion. In these two graphs the SCR data for the period of yawing were used for the analysis because the motion speeds were common to all of the motions. The graphs in the middle column are categorized into the robot motions "yawing" and "approach." The graphs in the right column are categorized into the speeds of the approach motion; in actuality the data of "fast" and "slow" are sufficient, but for reference the data of the yawing motion is included. The analysis of variance was performed for each case. There was a significant difference in Fig.4 with $\mathrm{P}<0.06$.

Fig.9 shows scatter plots of variations of SCR indices with respect to approach speeds. The variation was calculated as the amount under the fast approach motion minus the corresponding amount under the slow approach motion. The horizontal axis is scaled by variation of the SCR magnitude, and the vertical axis the SCR frequency. There is a negative correlation between them with $r=-0.62$. The approximated trendline is also shown in Fig.9.

Table II shows correlation coefficients between skin temperature of annular finger and SCR indices. The number marked with $*$ represents a correlation coefficient within the rejection region of the noncorrelation test with $\mathrm{P}<0.05$.

Factor analysis was carried out for the subjective ratings and two factors were extracted. The factor loadings are shown 
TABLE II

CORRELATION COEFFICIENTS BETWEEN SKIN TEMPERATURE OF ANNULAR FINGER AND SCR INDICES. ${ }^{*} P<0.05$

\begin{tabular}{|l|l|l|l|l|}
\cline { 2 - 5 } \multicolumn{1}{c|}{} & \multicolumn{2}{c|}{ SCR mag. } & \multicolumn{2}{c|}{ SCR freq. } \\
\cline { 2 - 5 } & yawing & appr. & yawing & appr. \\
\hline skin temperature & $0.58^{*}$ & 0.19 & 0.27 & $0.52^{*}$ \\
\hline
\end{tabular}

TABLE III

FACTOR LOADINGS WITH RESPECT TO THE SUBJECTIVE RATINGS

\begin{tabular}{c|rr}
\hline scale & Factor1 & Factor2 \\
\hline relaxed & 0.816 & -0.147 \\
pleasant & 0.573 & 0.094 \\
secure & 0.783 & 0.291 \\
far & - & -0.191 \\
slow & 0.141 & 0.990 \\
\hline
\end{tabular}

in Table III. Table IV shows correlation coefficients among each factor score, the closest distance between the robot and a subject, and the SCR indices. The numbers marked with $*$ (or $* *$ ) represents correlation coefficients within the rejection region of the noncorrelation test with $P<0.2$ (or $P<0.1$ ) respectively.

\section{DISCUSSION}

\section{A. SCR indices}

When the same stimuli are given repeatedly the EDA responses tend to be reduced gradually. This phenomenon is known as habituation. In this experiment similar robot motions were presented to a subject twice, therefore the habituation might be occurred. In Fig.2, comparing the graphs in the left column with those in the right reveals that under the yawing motion, which was the common motion in each measurement, the fluctuations of the SCR in the graphs in the right column were relatively smaller. As shown in Fig. 3 and Fig.6 the first measurements were relatively larger than the second measurements, but ANOVA revealed no significant difference between the two sets of measurements. Therefore we might say that there was no significant habituation in this experiment. There was a short interval when subjects filled out a questionnaire between successive robot motions and this interval might have attenuated the habituation.

TABLE IV

CORRELATION COEFFICIENTS BETWEEN FACTOR SCORES, THE CLOSEST DisTANCE AND SCR INDICES. ${ }^{*} P<0.20,{ }^{* *} P<0.10$

\begin{tabular}{|c|c|c|c|c|c|}
\cline { 3 - 6 } \multicolumn{1}{c|}{} & closest & \multicolumn{2}{c|}{ SCR mag. } & \multicolumn{2}{c|}{ SCR freq. } \\
\cline { 3 - 6 } \multicolumn{1}{c|}{} & dist. & yawing & appr. & yawing & appr. \\
\hline Factor 1 & -0.02 & -0.26 & -0.11 & 0.25 & -0.28 \\
\hline Factor 2 & $-0.55^{* *}$ & $0.44^{*}$ & $0.53^{* *}$ & -0.21 & $0.46^{*}$ \\
\hline dist. & 1 & 0.05 & -0.11 & 0.26 & -0.28 \\
\hline
\end{tabular}

It can be seen in Fig. 4 and Fig. 7 that both SCR indices under the approach motion were larger than those under the yawing motion, and these differences in SCR magnitude were statistically signficant (ANOVA, $P<0.06$ ). We could conclude that the approach motion tended to give stronger stimuli to the subject than the yawing motion at a distance.

Different tendencies were observed in Fig.5 and Fig.8. Namely the SCR magnitude under the fast motion tended to be larger than that under the slow motion, but SCR frequency indicated an opposite tendency against SCR magnitude. Furthermore ANOVA revealed no significant difference between them. The same feature was observed in Fig.9 and Fig.2. The inclination of the approximated line in Fig.9 shows negative correlations in the variations between SCR magnitude and frequency. As shown in Fig.2, the SCR wave whose magnitude was larger tended to have a smaller number of ripples. Conversely the SCR wave with an abundance of ripples tended to be smaller in magnitude. To summarize in terms of motion speed the difference in impression of robot motion might not be distinguishable. One possible cause might be that both robot speeds, "fast" and "slow", were not so large that levels of SCR waves remained in low response. From this we could say that in this experiment the factor of distance would be more crucial than that of speed.

We also measured the closest distance between a subject and the robot when the robot was proceeding on the step (3) in Fig.1. However no significant correlation was found in the SCR indices. The measured closest distances were around $45-65 \mathrm{~cm}$. It was speculated that its range was too narrow to be reflected in the SCR indices.

As shown in Table II there were significant correlations between skin temperatures and some SCR indices. It could be that some subjects whose skin temperatures were lower indicated less activity in the SCR to robot motions. It is recommended that the subjects should be maintained at normothermia before SCR measurements.

\section{B. Subjective rating}

In Table III, since all factor loadings of factor 1 were positive, factor 1 was considered a size factor. The loadings of factor 2 had both positive and negative values, therefore factor 2 was considered a shape factor.

In factor 1 the loadings with respect to "relaxed", "pleasant", and "secure" were larger. These adjectives represented the subjects' frame of mind and it was considered that factor 1 explained "relief of mind."

In factor 2 the loading with respect to "slow" was particularly large and the loading with respect to "far" was negative, which seems a bit strange. If a robot is close to a subject its motion should be felt quickly. In this experiment the closest distance between a subject and the robot was different in each experimental situation. Table $\mathrm{V}$ shows mean values and standard deviations of the closest distances categorized by 
TABLE V

MEAN VALUES AND STANDARD DEVIATIONS OF THE CLOSEST DISTANCES CATEGORIZED INTO THE MOTION SPEED

\begin{tabular}{|l||r|r|}
\hline motion speed & mean $(\mathrm{cm})$ & std.dev. \\
\hline fast & 57.5 & 6.0 \\
\hline slow & 53.8 & 5.8 \\
\hline
\end{tabular}

approach speed. The mean distance under the slow motion was smaller than that under the fast motion. This is consistent with the trend appearing in the loadings of factor 2 . Therefore the loading of factor 2 reflected the subjects' observations on robot motion. We might say that the factor 2 explained "observation on motion."

In Table IV factor 2 has correlations with the closest distance, the SCR magnitudes under both yawing motion and approach motion, and the SCR frequency under approach motion. As discussed in the previous paragraph the correlation between factor 2 and the closest distance was comprehensible. However the factor 2 was remarkable in that it had correlations with the SCR indices, but closest distance did not correlate so highly with SCR indices. We suggest that the SCR indices correlate not with factor 1 , which reflected frame of mind, but with factor 2, which reflected the subjects' observations on the robot motion.

\section{CONCLUSION AND FUTURE WORK}

In our previous psychophysiological investigations into impressions of robot motions, it was observed that blocking out either sound or sight attenuated EDA responses of subjects to the robot motion. One candidate for a rule of robot motion generation deduced from this result was that robots would have to reduce their motion speed in the immediate vicinity of humans because reducing their speed would result in reducing their noise as well as their movement. This rule was applied to a mobile robot and resulting human impressions were investigated in this paper.

Prepared robot movement was as follows: the robot searched for an object with a color equivalent to the skin using a video camera, started yawing at 30 degrees on the spot repeatedly, and then about 30 seconds later began to approach the subject at a specific speed of "slow" or "fast." During the experiment the SCR of the subject was measured. The subjects rated their impressions by means of the semantic difference technique. Both the magnitude and the frequency of the SCR were analyzed by ANOVA, and the subjective ratings were analyzed by factor analysis.

Consequently it was found that the approach motion tended to give stronger stimuli to the subject than the yawing motion at a distance. At the same time, while the robot was approaching the subject the difference in impressions between the "fast" motion and the "slow" motion might not be distinguishable. In this experiment the factor of the distance was more crucial than that of the speed. From the factor analysis two factors were extracted and interpreted as "relief of mind" and "observation on motion". An interesting result was obtained such that the SCR indices did not correlated with the factor of "relief of mind", but did with the factor of "observation on motion".

From the results of this experiment we cannot exactly assert that the slower movement of the mobile robot in the immediate vicinity of the subjects elicits lower SNS response as the rule proposes. In further experiments robot motions with several speeds should be tested. Alternatively, it was shown that the SCR responses to the approach motion were significantly different from the SCR responses to the motion in the distant area. To confirm this finding further investigations should be performed as well.

\section{REFERENCES}

[1] RIA/ANSI R15.06 - 1999, American National Standard for Insustrial Robots and Robot System - Safety Requirements, American Nathional Standards Institute, 1999

[2] B. Martínez-Salvador, M. Pérez-Francisco, and A.P. Del Pobil, Collision Detection between robot arms and people, J. of Intelligent and Robotic Systems, vol. 38, pp.105-119, 2003.

[3] B. Karlsson, N. Karlsson, and P.Wide, A dynamic safety system based on sensor fusion, J. of Intelligent Manufacturing, vo11. , pp.475-483, 2000.

[4] Jozef Zurada, Andrew L. Wright, and James H. Graham, A NeuroFuzzy Approach for Robot System Safety, IEEE Trans. on Systems, Man, and Cybernetics-Part C, Vol.31, No.1, pp.49-64, 2001

[5] Y. Yamada, Y. Hirasawa, S. Huang, Y. Umetani, and S. Kazutsugu, Human-Robot Contact in the Safeguarding Space, IEEE/ASME Trans. on Mechatronics, vol. 2, no. 4, pp.230-236, 1997.

[6] H. O. Lim and K. Tanie, Collision-Tolerant Control of Human-Friendly Robot With Viscoelastic Trunk, IEEE/ASME Trans. on Mechatronics, vol. 4, no. 4, pp.417-427, 1999.

[7] M. Nokata, K. Ikuta and H. Ishii, Safety-optimizing Method of Humancare Robot Design and Control, Proc. of the 2002 IEEE International Conference on Robotics \& Automation, pp.1991-1966, 2002.

[8] D. Kulic and E.Croft, Safe Planning for Human Robot Interaction, Proc. of the Int. Conf. on Robotics and Automation 2004.

[9] S. Shibata and H. Inooka, Emotional evaluations on robot motions by using rating scale method, Human Eng., vol. 31, no.2, pp.151-159, 1995. (In Japanese)

[10] R. Ikeura, H. Ootsuka, and H. Inooka, Study on emotional evaluation of robot motions based on galvanic skin reflex, Human Eng., vol. 31, no.5, pp.355-358, 1995. (In Japanese)

[11] Y. Yamada, Y. Umetani, and Y. Hirasawa, Proposal of a Psychophysiological Experiment System Applying the Reaction of Human Pupillary Dilation to Frightening Robot Motion, Proc. of 1999 IEEE Int. Conf. on SMC, vol. II, pp.1052-1057, 1999.

[12] N. Hanajima, M. Fujimoto, M. Yamashita, and H. Hikita, On influence of the actuation noise on the impressions of robot's reaching motions, Proc. of HIS2002 (In Japanese), 2002.

[13] N. Hanajima, M. Fujimoto, H. Hikita, and M. Yamashita, "Influence of Auditory and Visual modalities on Skin Potential Response to Robot Motions", Proc. of 2004 IEEE/RSJ International Conference on Intelligent Robots and Systems, 2004, 1226 - 1231

[14] M. E. Dawson, A. M. Schell, and D. L. Filion, The electrodermal system, Handbook of Psychophysiology 2nd ed. Cambridge University Press, 2000.

[15] S-PLUS 2000 Guide to Statistics, Data Analysis Products Division, MathSoft, Inc. Seattle, Washington, 1999 